\section{VÂNia Muniz Néquer Soares ${ }^{1}$ \\ Kleyde Ventura de Souza ${ }^{2}$}

Elbens Marcos Minoreu de AzeVedo ${ }^{3}$

Carla Rejane Possebon ${ }^{4}$

Fernanda FerRerRa MARQues ${ }^{5}$

Artigo Original

Palavras-chave

Mortalidade materna

Saúde materna

Gravidez de alto risco

Pesquisa sobre serviços de saúde

Assistência hospitalar

Keywords

Maternal mortality

Maternal welfare

Pregnancy, high-risk

Health services research

Hospital care

Correspondência

Kleyde Ventura de Souza

Escola de Enfermagem da UFMG

Avenida Professor Alfredo Balena, 190 - Sala Santa Efigênio

(EP: $30130-100$

Belo Horizonte (MG), Brasil

Recebido

$25 / 05 / 2012$

\title{
Causas de mortalidade materna segundo níveis de complexidade hospitalar
}

\author{
Causes of maternal mortality according to levels of \\ hospital complexity
}

\section{Resumo}

OBJETIVOS: Identificar e analisar as causas da mortalidade materna, segundo os níveis de complexidade hospitalar. MÉTODOS: Estudo quantitativo, descritivo e de corte transversal das mortes maternas hospitalares ocorridas no Paraná, Brasil, nos triênios de 2005-2007 e 2008-20 10. Foram utilizados dados dos estudos de casos de óbitos maternos, que foram elaborados pelo Comitê Estadual de Prevenção da Mortalidade Materna. As variáveis estudadas foram o local e as causas dos óbitos, a transferência hospitalar e a evitabilidade. Foram calculadas a razão de mortalidade materna, a proporção e a taxa de letalidade hospitalar, segundo os subgrupos hospitais de referência para gestações de alto e baixo risco. RESULTADOS: A razão de mortalidade materna, incluindo os óbitos maternos tardios, foi 65,9/100.000 nascidos vivos (de 2008 a 2010). O local do óbito foi o hospital em cerca de $90 \%$ dos casos, em ambos os períodos. No primeiro triênio, nos hospitais de referência para gestação de alto risco, a taxa de letalidade hospitalar foi de 158,4/100.000 partos e, no segundo, 132,5/100.000 e as principais causas foram: pré-eclampsia/eclampsia, infecção urinária, infecção puerperal e causas indiretas. Nos hospitais de referência para gestação de baixo risco, as taxas de letalidade hospitalar foram: 76,2/100.000 e 80,0/100.000, e como principais causas: hemorragias, embolias e complicações anestésicas. Em 64 (2005-2007) e 71\% (2008-2010) dos casos, o óbito ocorreu no hospital do internamento inicial. Foram considerados evitáveis $90 \%$ dos óbitos no segundo triênio. CONCLUSÕES: Há dificuldades no atendimento das complicações obstétricas em ambos os níveis de atenção de baixa e alta complexidade. A capacitação dos profissionais para o atendimento às emergências obstétricas e o monitoramento do uso dos protocolos em todos os níveis hospitalares deve ser priorizada para a redução das mortes maternas evitáveis.

\section{Abstract}

PURPOSES: To identify and to analyze maternal mortality causes, according to hospital complexity levels. METHODS: A descriptive-quantitative cross-sectional study of maternal deaths that occurred in hospitals in Paraná, Brazil, during the periods from 2005 to 2007 and from 2008 to 2010 . Data from case studies of maternal mortality, obtained by the State Committee for Maternal Mortality Prevention, were utilized. The study focused on variables such as site and causes of death, hospital transfer, and avoidability. Maternal mortality rate, proportions, and hospital lethality ratio were calculated according to subgroups of low and high-risk pregnancy reference hospitals. RESULTS: Maternal mortality rate, including late maternal deaths, was 65.9 per 100.000 live-borns (from 2008 to 2010). Almost 90\% of all maternal deaths occurred in the hospital environment, in both periods. The hospital lethality ratio at the high-risk pregnancy reference hospital was 158.4 deaths per 100,000 deliveries during the first period and 132.5/100,000 during the second, and the main causes were pre-eclampsia/eclampsia, puerperal infection, urinary tract infection, and indirect causes. At the low-risk pregnancy reference hospitals, the hospital lethality ratios were 76.2/100,000 and 80.0/100,000, and the main causes of death were hemorrhage, embolism, and anesthesia complications. In 64 (2005-2007) and in 71\% (2008-2010) of the cases, the patients died in the same hospital of admission. During the second period, $90 \%$ of the casualties were avoidable. CONCLUSIONS: Hospitals of both levels of complexity are having difficulties in treating obstetric complications. Professional training for obstetric emergency assistance and the monitoring of protocols at all hospital levels should be considered by the managers as a priority strategy to reduce avoidable maternal deaths.

\section{Trabalho realizado na Universidade Tuiuti do Paraná - UTP - Curitiba (PR), Brasil.}

'Departamento de Enfermagem da Faculdade de Ciências Biológicas e da Saúde, Universidade Tuiuti do Paraná - UTP - Curitiba (PR), Brasil. 2Departamento Materno-Infantil e de Saúde Pública da Escola de Enfermagem, Universidade Federal de Minas Gerais - UFMG - Belo Horizonte (MG), Brasil.

${ }^{3}$ Departamento de Medicina, Universidade Estadual de Londrina - UEL - Londrina (PR), Brasil.

${ }^{4}$ Hospital de Clínicas, Universidade Federal do Paraná - UFPR - Curitiba (PR), Brasil.

${ }^{5}$ Secretaria Municipal de Saúde de Paranaguá - Paranaguá (PR), Brasil.

Conflitos de interesses: não há. 


\section{Introdução}

A razão de mortalidade materna (RMM) é um indicador de desenvolvimento humano, econômico, social e da qualidade de assistência à saúde. A ocorrência de óbitos maternos evitáveis reflete as precárias condições econômicas, culturais e tecnológicas de um país ou de uma sociedade e constitui-se numa violação dos direitos reprodutivos das mulheres, tratando-se, portanto, de uma preocupação mundial ${ }^{1}$.

Recentes estimativas projetaram a ocorrência de aproximadamente 273.000 mortes maternas, em todo o mundo, em $2011^{2}$. No entanto, o declínio global da RMM tem sido lento, aproximadamente 2,3\% ao ano, desde 1990 . Em 2008, estimava-se a ocorrência de 358.000 mortes maternas no mundo; em 1990, eram 546.000. As taxas passaram de 320 por 100.000 nascidos vivos (nv), em 1990, para 260 por 100.000 nv, em 2008. A RMM, em 2008, foi maior nas regiões em desenvolvimento (290) em contraste com as desenvolvidas (14). Na América Latina e no Caribe, a taxa estimada em 2008 foi de 85/100.000 nv e, no Brasil, 58/100.000 nv, com 1.800 óbitos anuais ${ }^{3}$.

No entanto, a maioria de tais óbitos poderia ser evitada se as condições de saúde locais fossem semelhantes àquelas dos países desenvolvidos, com acesso a serviços de referência de qualidade ${ }^{1,4,5}$. Por outro lado, em alguns países pouco desenvolvidos, com situação econômica desfavorável, tais como Chile, Cuba e Costa Rica, a RMM é substancialmente inferior a de países em desenvolvimento, como é o Brasil, demonstrando que a morte materna pode ser um indicador da decisão política de garantir a saúde a esta parcela da população $0^{6,7}$.

No Brasil, dois fatores dificultam o real monitoramento do nível e da tendência da mortalidade materna: a subinformação e o sub-registro das declarações das causas de óbito ${ }^{7,8}$. No Paraná, a subnotificação vem sendo corrigida desde 1990 pelos Comitês de Prevenção da Mortalidade Materna, que atuam junto às secretarias municipais e de estado da saúde, cujas vigilâncias epidemiológicas, em todo o estado, investigam os óbitos de mulheres em idade fértil, identificando os óbitos maternos?

Apesar da redução da mortalidade global verificada na última década, identificou-se a necessidade de revisar estratégias de ação para concentrar esforços nas áreas que apresentam maior mortalidade materna, como no caso de países como o Brasil, que deverá apresentar RMM igual ou inferior a 35 óbitos por 100.000 nv até 2015 para alcançar o quinto objetivo de desenvolvimento do milênio, que foi estabelecido pela Organização das Nações Unidas $(\mathrm{ONU})^{10,11}$.

Para prevenir as mortes maternas são necessárias medidas para melhoria da formação dos profissionais que prestam assistência às gestantes, parturientes e puérperas à garantia de um parto seguro, além da organização de um sistema de referência para atendimento eficiente às emergências obstétricas, dentre outras. São imprescindíveis ações amplas e articuladas que visem uma mudança efetiva do atual modelo assistencial na atenção obstétrica, haja vista as inegáveis deficiências evidenciadas e considerando o elevado número de óbitos evitáveis ${ }^{12,13}$.

Faz-se necessário ampliar o conhecimento sobre a mortalidade materna, utilizando-se os dados disponíveis para melhor analisar as causas dos óbitos e, desta maneira, realizar uma melhor avaliação da preocupante situação atual, apontando sugestões para sua efetiva redução ${ }^{5}$.

Nessa perspectiva, este estudo buscou identificar e analisar as causas da mortalidade materna, segundo níveis de complexidade hospitalar, haja vista que aproximadamente $90 \%$ dos nascimentos e dos óbitos maternos no estado do Paraná, Brasil, ocorre em hospitais. Espera-se que este estudo possa contribuir como ponto de partida para o desenvolvimento de outras investigações.

\section{Métodos}

Estudo quantitativo, descritivo e de corte transversal, que visou conhecer as taxas e causas da mortalidade materna no estado do Paraná, segundo o nível de complexidade hospitalar (hospital de referência para gestações de baixo e alto risco), comparando-se dois triênios, 2005-2007 e 2008-2010.

Para classificação hospitalar, obteve-se a listagem das maternidades ou hospitais, classificados como hospitais de referência para gestação de alto risco, junto à Secretaria Estadual de Saúde, sendo considerado para este estudo apenas os hospitais secundários e terciários que contavam com serviço de unidade de tratamento intensivo (UTI) adulto e/ou neonatal (29 hospitais nos dois triênios). Tais hospitais passaram a constituir o grupo dos hospitais de referência para gestação de alto risco. Os demais 298 hospitais no triênio 2005-2007 e os 308 no triênio 2008-2010 compuseram o grupo de hospitais de referência para gestação de baixo risco. Todos eram credenciados pelo Sistema Único de Saúde (SUS).

Para obtenção dos dados sobre os óbitos maternos, utilizaram-se os Estudos de Caso de Óbitos Maternos elaborados pelo Comitê Estadual de Prevenção da Mortalidade Materna (CEPMM-PR). Esses estudos de caso foram elaborados a partir dos inquéritos epidemiológicos dos óbitos maternos, realizados pelas equipes das vigilâncias epidemiológicas dos municípios, agrupadas nas 22 regionais de saúde do estado do Paraná.

Após tabulação dos dados, foram calculadas e analisadas para cada um dos triênios as RMM do estado (número de óbitos maternos divididos pelo de nv versus $100.000 \mathrm{nv}$ ). Posteriormente, calcularam-se, para cada um dos triênios e de acordo com o grau de complexidade hospitalar, a frequência e os percentuais de óbitos maternos, segundo o local do óbito, a necessidade de transferência e evitabilidade do óbito 
e a TLM hospitalar (número de óbitos maternos divididos pelo número de partos hospitalares versus 100.000 partos).

A análise estatística dos dados ocorreu da seguinte forma: para comparação de hospitais ou triênios, em relação às taxas, adotou-se o modelo de Poisson para o número de ocorrências, considerando-se as medidas de exposição associadas a cada caso. Intervalos com $95 \%$ de confiança foram construídos para as razões entre taxas. Para comparação dos hospitais e triênios em relação à probabilidade de transferência, foi considerado o teste exato de Fisher. Para comparação dos hospitais e triênios, em relação à homogeneidade das classificações de evitabilidade, utilizou-se o teste do $X^{2}$. Valores de p menores que 0,05 indicaram significância estatística. Adotaramse, neste estudo, as seguintes definições da Organização Mundial de Saúde (OMS) ${ }^{14}$ :

- morte materna: é a morte de uma mulher durante a gestação ou dentro de um período de 42 dias, após o término da gestação, independente da duração ou da localização da gravidez, devido à qualquer causa relacionada com ou agravada pela gravidez ou por medidas em relação a ela, porém não devido a causas acidentais ou incidentais;

- morte materna tardia: é a morte de uma mulher por causas obstétricas diretas ou indiretas, com mais de 42 dias, porém menos de um ano após o término da gravidez;

- morte obstétrica direta: resultante de complicações obstétricas na gravidez, no parto e no puerpério, devido a intervenções, omissões, tratamento incorreto ou uma cadeia de eventos resultantes de qualquer das causas mencionadas;

- morte obstétrica indireta: resultante de doenças existentes antes da gravidez ou que se desenvolveram durante a gravidez, não devido a causas obstétricas diretas, mas que foram agravadas pelos efeitos fisiológicos da gravidez.

Para os casos em que não foi possível determinar a causa da morte materna, a mesma foi classificada como indeterminada.
O projeto de pesquisa foi aprovado pelo Comitê de Ética em Pesquisa em Seres Humanos, protocolo 460/2012.

\section{Resultados}

Ocorreram no triênio 2005-2007, 461.999 nascimentos (nv) e 341 óbitos maternos, incluindo os tardios, determinando uma RMM de 73,8/100.000 nv. No triênio seguinte (2008-2010), ocorreram 452.444 nascimentos (nv) e 298 óbitos maternos, ou seja, uma RMM de 65,9/100.000 nv, verificando-se uma redução não significante de $4,8 \%$ em relação ao triênio anterior $(\mathrm{p}=0,607)$. Excluindo-se os óbitos tardios contatou-se uma RMM em torno de 60/100.000 nv (Tabela 1).

Nos dois períodos, predominaram as causas obstétricas diretas, RMM de 42/100.000 nv, duas vezes superior à RMM das causas obstétricas indiretas (18 e 16/100.000 nv em cada período). Observou-se redução significativa apenas nas mortes maternas tardias (Tabela 1).

$\mathrm{Na}$ Tabela 2, pode-se observar que de 2005 a 2007, ocorreram 310 (90,9\%) óbitos maternos em ambiente hospitalar e $31(9,1 \%)$ em domicílio ou via pública. No triênio de 2008-2010, foram 275 (92,3\%) e 18 (6,0\%), respectivamente. Nesse segundo período, em cinco casos $(1,7 \%)$ o local do óbito foi ignorado. No primeiro triênio, dos 310 óbitos maternos hospitalares, 174 (51\%) ocorreram em um dos hospitais de referência para gestação de alto risco e $136(39,9 \%)$ naqueles de baixo risco. No segundo triênio, das 275 mortes, 139 (47\%) ocorreram em instituições classificadas como de referência para gestação de alto risco e $136(45,3 \%)$ nas consideradas de baixo risco.

A Tabela 3 mostra a distribuição dos óbitos maternos segundo as causas das mortes e o nível de referência hospitalar no triênio 2005-2007, em que, nos hospitais de referência para gestação de alto risco, ocorrem 109.869 partos (38\% do total de partos hospitalares) e 174 óbitos, com taxa de letalidade materna (TLM) de 158,4/100.000 partos, e, nos hospitais de referência para gestação de baixo risco, ocorreram 178.469 partos (62\% do total) e 136 óbitos, com TLM de 76,2/100.000 partos. Em tal triênio, os hospitais de referência para gestação de alto risco

Tabela 1. Razão de mortalidade materna no Paraná nos triênios 2005-2007 e 2008-2010

\begin{tabular}{|c|c|c|c|c|c|c|c|c|}
\hline \multirow{2}{*}{ Mortes maternas } & \multicolumn{2}{|c|}{$2005-2007$} & \multicolumn{2}{|c|}{$2008-2010$} & \multirow{2}{*}{$\begin{array}{l}\text { \% de reduç̃óo da RMM } \\
\text { entre dois triênios }\end{array}$} & \multirow{2}{*}{$\begin{array}{l}\text { Razão de } \\
\text { RMM** }^{*}\end{array}$} & \multirow{2}{*}{ IC95\% } & \multirow{2}{*}{ Valor $p$} \\
\hline & $n$ & RMM* & $n$ & RMM* & & & & \\
\hline Obstétricas diretas & 197 & 42,6 & 191 & 42,2 & $-1,0$ & 1,01 & $0,82-1,23$ & 0,9 \\
\hline Causa indeterminada & 9 & 1,9 & 6 & 1,3 & $-46,9$ & 1,46 & $0,46-4,51$ & 0,6 \\
\hline Total de mortalidade materna não tardia & 289 & 62,6 & 270 & 59,7 & $-4,8$ & 1,04 & $0,88-1,23$ & 0,6 \\
\hline Mortalidade materna tardia & 52 & 11,3 & 28 & 6,2 & $-81,9$ & 1,81 & $1,12-2,93$ & 0,01 \\
\hline
\end{tabular}

RMM: razão de mortalidade materna; *RMM por 100.000 nascidos vivos; **RMM do período de 2005 a 2008 em relação àquela do período de 2009 a 2010 ; IC95\%: intervalo de confiança de $95 \%$ 
apresentaram as causas mais frequentes de mortes maternas, dentre as obstétricas diretas, em ordem decrescente: pré-eclampsia/eclampsia (28 casos), infecção urinária (15), infecção puerperal (12) e aborto (12). Já nos hospitais de referência para gestação de baixo risco, observaram-se as seguintes causas: hemorragias (21), embolias (12) e complicações anestésicas (8). O número de óbitos por causas obstétricas indiretas (48) foi mais elevado nos hospitais de referência para gestação de alto risco, enquanto os óbitos maternos tardios (25) foram mais frequentes naqueles para gestação de baixo risco (Tabela 3).

A Tabela 4 mostra distribuição dos óbitos maternos segundo as causas das mortes e o nível de referência hospitalar no triênio 2008-2010. Em tal triênio, foram realizados nos hospitais de referência para gestação de alto risco 104.658 partos (38\% do total), e ocorreram 139 óbitos maternos, com TLM de 132,5/100.000 partos. Nos hospitais de referência para gestação de baixo risco,

Tabela 2. Número e percentual de óbitos maternos segundo o local do óbito, nos triênios 2005-2007 e 2008-2010

\begin{tabular}{|c|c|c|c|c|c|}
\hline \multirow{2}{*}{ Local do óbito } & \multicolumn{2}{|c|}{ 2005-2007 } & \multicolumn{2}{|c|}{ 2008-2010 } & \multirow{2}{*}{ Valor $\mathrm{p}$} \\
\hline & $n$ & $\%$ & $\mathrm{n}$ & $\%$ & \\
\hline $\begin{array}{l}\text { Hospital de referência para } \\
\text { gestação de baixo risco }\end{array}$ & 136 & 39,9 & 136 & 45,3 & 0,1 \\
\hline $\begin{array}{l}\text { Hospital de referência para } \\
\text { gestação de alto risco }\end{array}$ & 174 & 51,0 & 139 & 47,0 & 0,3 \\
\hline Subtotal & 310 & 90,9 & 275 & 92,3 & \\
\hline Domicílio & 26 & 7,6 & 11 & 3,7 & - \\
\hline Via pública & 5 & 1,5 & 7 & 2,3 & - \\
\hline Ignorado & 0 & 0 & 5 & 1,7 & - \\
\hline Total & 341 & 100 & 298 & 100 & \\
\hline
\end{tabular}

realizaram-se 169.784 partos (62\% do total), e ocorreram 136 óbitos maternos, com TLM de 80,0/100.000 (Tabela 4). Nesse triênio, nos hospitais de referência para gestação de alto risco as mesmas causas predominaram, com alguma inversão de ordem: pré-eclampsia/eclampsia (24), infecção puerperal (13) passou para a segunda causa e a infecção urinária (11), a terceira. Nos hospitais de referência para gestação de baixo risco, as principais causas de morte em ordem decrescente foram: hemorragias (24), embolias (9), complicações anestésicas (9) e aborto (8). As causas obstétricas indiretas tiveram frequência semelhante nos dois grupos de hospitais neste triênio (33 e 32 óbitos, respectivamente), e os óbitos maternos tardios (13) foram mais elevados nos hospitais de referência para o alto risco.

A análise de cada um dos triênios possibilitou a avaliação das TLM, segundo cada causa de óbito materno.

No triênio 2005-2007, nos hospitais de referência para gestação de alto risco a TLM para eclampsia foi de $25,5 / 100.000$ partos; naqueles para baixo risco esta taxa foi de 11,2. A razão entre as taxas foi de 2,2, indicando que a razão nos hospitais de referência para o alto risco foi $127,4 \%$ superior àquela observada nos de baixo risco $(\mathrm{p}=0,007)$. Para hemorragia, a TLM foi de 17,3/100.000 partos no grupo de hospitais de referência para gestação de alto risco; e nos de baixo risco, de 11,8. A razão entre as taxas foi de 1,4 , indicando que a taxa nos hospitais de alto risco foi $47,0 \%(\mathrm{p}=0,2)$ superior, quando comparada àquela dos hospitais de referência para o baixo risco.

Para a infecção puerperal, no mesmo triênio, a TLM foi de 10,9/100.000 partos nos hospitais de referência para gestação de alto risco, e, nos de baixo risco, 3,4/100.000 partos. A razão entre as taxas foi de 3,2, indicando que a

Tabela 3. Distribuição dos óbitos maternos segundo as causas das mortes e o nível de referência hospitalar no triênio 2005-2007

\begin{tabular}{|c|c|c|c|c|c|c|c|c|c|}
\hline \multirow{3}{*}{ Causas } & \multicolumn{4}{|c|}{ Hospital de referência à gestação } & \multirow{2}{*}{\multicolumn{2}{|c|}{ Total }} & \multirow{3}{*}{ Razão de taxas } & \multirow{3}{*}{ IC95\% } & \multirow{3}{*}{ Valor $\mathrm{p}$} \\
\hline & \multicolumn{2}{|c|}{ Alto risco } & \multicolumn{2}{|c|}{ Baixo risco } & & & & & \\
\hline & n & $\begin{array}{c}\text { Taxa de } \\
\text { lefalidade }\end{array}$ & n & $\begin{array}{c}\text { Taxa de } \\
\text { lefalidade }\end{array}$ & n & $\begin{array}{c}\text { Taxa de } \\
\text { letalidade }\end{array}$ & & & \\
\hline Obstétricas direfas & 108 & 98,3 & 82 & 45,9 & 190 & 65,9 & 2,1 & $0,59-2,86$ & $<0,001$ \\
\hline Aborto & 12 & 10,9 & 5 & 2,8 & 17 & 5,9 & 3,8 & $1,27-13,03$ & 0,01 \\
\hline Pré-eclampsia, eclampsia & 28 & 25,5 & 20 & 11,2 & 48 & 16,6 & 2,2 & $1,23-4,11$ & 0,007 \\
\hline Hemorragia & 19 & 17,3 & 21 & 11,8 & 40 & 13,9 & 1,4 & $0,74-2,72$ & 0,2 \\
\hline Complicações anestésicas & 2 & 1,8 & 8 & 4,5 & 10 & 3,5 & 0,4 & $0,04-1,35$ & 0,4 \\
\hline Infecção puerperal & 12 & 10,9 & 6 & 3,4 & 18 & 6,2 & 3,2 & $1,12-9,73$ & 0,02 \\
\hline Embolia & 8 & 7,3 & 12 & 6,7 & 20 & 6,9 & 1,0 & $0,38-2,56$ & $\cdot$ \\
\hline Infecção urinária & 15 & 13,7 & 3 & 1,7 & 18 & 6,2 & 8,1 & $2,29-41,03$ & $<0,001$ \\
\hline Outras diretas & 12 & 10,9 & 7 & 3,9 & 19 & 6,6 & 2,7 & $1,01-7,70$ & 0,04 \\
\hline Obstétrica indireta & 48 & 43,7 & 26 & 14,6 & 74 & 25,7 & 2,9 & $1,82-4,93$ & $<0,001$ \\
\hline Óbito materno não especificado & 3 & 2,7 & 3 & 1,7 & 6 & 2,1 & 1,6 & $0,21-9,09$ & 0,8 \\
\hline Óbito materno fardio & 15 & 13,7 & 25 & 14,0 & 40 & 13,9 & 0,9 & $0,47-1,80$ & - \\
\hline Total & 174 & 158,4 & 136 & 76,2 & 310 & 107,5 & 2,07 & $1,65-2,60$ & $<0,001$ \\
\hline
\end{tabular}

IC95\%: intervalo de confiança de 95\%; total de partos hospitalares: 288.338 ; total de partos em hospitais referentes à gestação de alto risco: 109.869 ; número de partos em hospitais referentes à gestação de baixo risco: 178.469. Fonte: SIHSUS. 
taxa nos hospitais de referência para alto risco foi $224,9 \%$ superior àquela observada nos hospitais de referência para gestação de baixo risco, apresentando diferença significativa entre os dois grupos $(\mathrm{p}=0,02)$.

O aborto foi a quinta causa de óbito obstétrico direto neste triênio. A TLM foi de 10,9/100.000 partos nos hospitais de referência para gestação de alto risco, e 2,8/100.000 partos, de baixo risco. A razão entre as taxas foi de 3,8 ou $289,9 \%$ ( $\mathrm{p}=0,01)$ superior nos hospitais de referência para gestação de alto risco.

Para infecção urinária, a TLM foi de 13,7/100.000 partos nos hospitais de referência para gestação de alto risco, 8,1 vezes superior à taxa verificada nos hospitais de referência para gestação de baixo risco, cuja taxa foi de 1,7 $(\mathrm{p}<0,001)$ ou $722,2 \%$ superior. Para embolia, a TLM foi de 7,3/100.000 partos nos hospitais de referência para gestação de alto risco e 6,7/100.000 partos em hospitais de referência para gestação de baixo risco. Portanto, para esta variável não houve evidência de diferença entre os dois grupos de hospitais.
As TLM foram superiores nos hospitais de referência para gestação de baixo risco apenas para as complicações anestésicas e para os óbitos maternos tardios, mas não houve diferença estatisticamente significativa nestes casos.

No triênio 2008-2010, as TLM permaneceram mais elevadas nos hospitais de referência para gestação de alto risco para a maioria das causas, com diferença significativa em relação às taxas dos hospitais de referência para gestação de baixo risco nos casos de infecção urinária $(p=0,006)$ e infecção puerperal $(\mathrm{p}=0,049)$. O aborto foi a quarta causa de óbito no segundo triênio, sendo mais frequente nos hospitais de referência para gestação de baixo risco, mas com letalidade 1,2 vezes mais elevada nos hospitais de referência para gestação de alto risco. Nos hospitais de referência para gestação de baixo risco, as taxas foram mais elevadas para as complicações anestésicas e para embolia.

A Tabela 5 revela que, no primeiro triênio, para aproximadamente $64 \%$ dos casos não ocorreu transferência hospitalar ou o óbito ocorreu no hospital do internamento

Tabela 4. Distribuição dos óbitos maternos segundo as causas das mortes e o nível de referência hospitalar no triênio 2008-2010

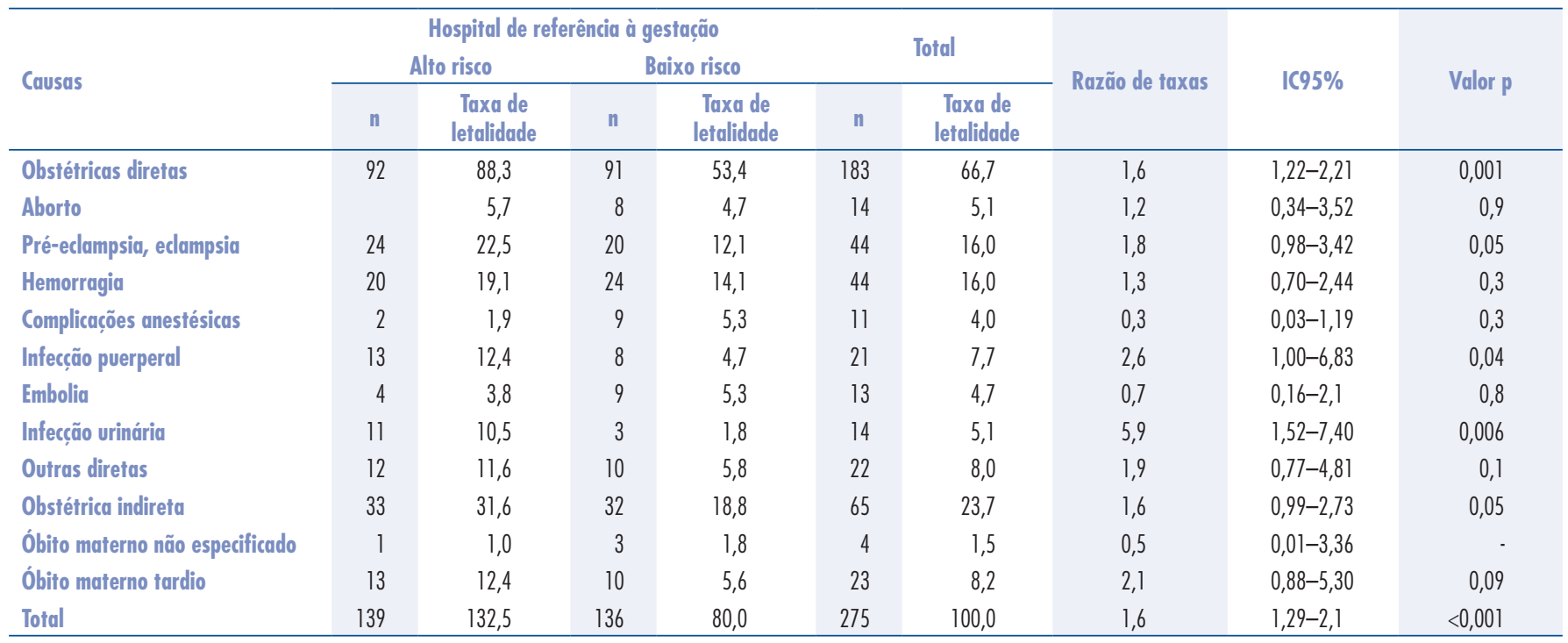

IC95\%: intervalo de confiança de 95\%; total de partos hospitalares: 274.442; total de partos em hospitais referentes à gestação de alto risco: 104.658; número de partos em hospitais referentes à gestação de baixo risco: 169.784. Fonte: SIHSUS.

Tabela 5. Percentual de transferências hospitalares e óbitos maternos evitáveis segundo o nível de referência hospitalar nos triênios 2005-2007 e 2008-2010

\begin{tabular}{|c|c|c|c|c|c|c|c|c|c|c|c|c|c|}
\hline \multirow{3}{*}{ Variável } & \multirow{3}{*}{ Classificação } & \multicolumn{4}{|c|}{ 2005-2007 } & \multirow{3}{*}{ Total } & \multirow{3}{*}{$\%$} & \multicolumn{4}{|c|}{ 2008-2010 } & \multirow{3}{*}{ Total } & \multirow{3}{*}{$\%$} \\
\hline & & \multicolumn{2}{|c|}{$\begin{array}{l}\text { Hospitais de referência } \\
\text { de alto risco }\end{array}$} & \multicolumn{2}{|c|}{$\begin{array}{c}\text { Hospitais de referência } \\
\text { de baixo risco }\end{array}$} & & & \multicolumn{2}{|c|}{$\begin{array}{l}\text { Hospitais de referência } \\
\text { de alto risco }\end{array}$} & \multicolumn{2}{|c|}{$\begin{array}{l}\text { Hospitais de referência } \\
\text { de baixo risco }\end{array}$} & & \\
\hline & & $n$ & $\%$ & $n$ & $\%$ & & & $n$ & $\%$ & $n$ & $\%$ & & \\
\hline \multirow[t]{2}{*}{ Transferência } & Não & 110 & 63,2 & 89 & 65,4 & 199 & 64,2 & 94 & 67,6 & 103 & 75,7 & 197 & 71,6 \\
\hline & Total & 174 & 100 & 136 & 100 & 310 & 100 & 139 & 100,0 & 136 & 100 & 275 & 100 \\
\hline \multirow{2}{*}{ Evitabilidade } & Inc. & 5 & 2,9 & 8 & 5,9 & 13 & 4,2 & 7 & 5,0 & 0 & 0,0 & 7 & 2,5 \\
\hline & Total & 174 & 100 & 136 & 100 & 310 & 100 & 139 & 100,0 & 136 & 100 & 275 & 100 \\
\hline
\end{tabular}

Inc.: Inconclusivo. 
inicial. No segundo triênio, 71,6\% dos óbitos ocorreram no mesmo hospital em que ocorreu o internamento.

Nos dois períodos não houve diferença significativa entre hospitais de referência para gestações de baixo e alto risco em relação à probabilidade de transferência (2005-2007, $\mathrm{p}=0,721 ; 2008-2010, \mathrm{p}=0,143)$.

Com relação à evitabilidade, a Tabela 5 demonstra que, no primeiro triênio, cerca de $80 \%$ dos óbitos maternos eram evitáveis em ambos os níveis de atenção hospitalar, e no segundo triênio os casos que seriam evitáveis aumentou para aproximadamente $90 \%$, segundo a avaliação dos Comitês de Prevenção da Mortalidade Materna do Paraná.

Nos dois períodos não houve diferença significativa entre hospitais de referência para gestações de baixo e alto risco, em relação à evitabilidade dos óbitos (2005-2007, $\mathrm{p}=0,4 ; 2008-2010, \mathrm{p}=0,5)$.

\section{Discussão}

O estado do Paraná apresenta RMM ainda elevada, próxima a 60/100.000 nv, se forem excluídos os óbitos maternos tardios, quando a taxa recomendada é próxima a 20/100.000 nv, conforme estabelece a OMS ${ }^{14}$.

Com relação à distribuição dos óbitos maternos segundo o local de ocorrência do óbito, evidenciou-se que cerca de 90\% deles ocorreram em hospitais. Estudo realizado no município de Recife (PE), com RMM 65/100.000 nv de 1999 a 2000, identificou que cerca de $80 \%$ dos óbitos ocorreram em hospitais públicos, filantrópicos ou universitários ${ }^{15}$, proporção inferior à encontrada neste estudo.

Considerando que, em média, no Brasil, 98\% dos partos ocorrem em ambiente hospitalar e em maternidades, deve-se voltar atenção, sobretudo, à assistência médica e hospitalar e às complicações obstétricas, que são na sua maioria evitáveis, necessitando-se assim da avaliação dos determinantes dessas mortes, para que as autoridades sanitárias, os profissionais, os serviços e os centros formadores de profissionais promovam medidas para qualificação da assistência ${ }^{5,11,12,16,17}$.

Com relação às causas das mortes maternas, identificou-se um padrão semelhante nos dois triênios estudados com a predominância dos óbitos por pré-eclampsia/eclampsia, infecções urinária e puerperal, dentre as causas obstétricas diretas, nos hospitais de referência para gestação de alto risco e a predominância dos óbitos por hemorragia, embolia e complicações anestésicas naqueles para gestação de baixo risco. Reitera-se, portanto, a capacidade limitada de prevenção da mortalidade pelo sistema de saúde ${ }^{18}$.

Pesquisas têm demonstrado o risco elevado relativo às mortes maternas para o parto cesárea, em relação ao vaginal, principalmente associado à ocorrência de hemorrágicas, infecciosas, embolia pulmonar e acidentes anestésicos associados ao parto cesárea, em relação ao vaginal ${ }^{19}$. Tais fatos indicam, por conseguinte, a premente necessidade de implementação de estratégias mais efetivas, como o acesso a serviços de qualidade e em tempo oportuno, a necessidade da melhoria da assistência ao parto normal e a redução de cesarianas desnecessárias, bem como de qualificação profissional para identificação e tratamento adequados aos casos de risco e às urgências/emergências obstétricas ${ }^{20}$.

Em estudo realizado no Hospital da Universidade Federal de Minas Gerais (UFMG), que é referência para todo o estado, em uma série histórica de 75 anos (1927-2001) sobre as taxas de mortalidade, observou-se nos últimos 15 anos uma taxa estabilizada em torno de 46/100.000 nv ${ }^{21}$. Em outro estudo, realizado no Hospital das Clínicas de Porto Alegre, que é referência para o estado do Rio Grande do Sul, verificou-se que, entre 1980 e 1999, a taxa se manteve estável, entre 90,0 e 109,0/100.00018. Estas taxas se mostram inferiores às encontradas no atual estudo para o grupo de hospitais de referência para gestação de alto risco, que indica a urgente necessidade de avaliação e monitoramento da assistência prestada, particularmente, nesse grupo de hospitais ${ }^{12}$.

A TLM total nos hospitais de referência para gestação de alto risco (158/100.000 e 132,5/100.000 partos, respectivamente) foi cerca de duas vezes superior às taxas dos hospitais de referência para o baixo risco $(76,2 / 100.000 \mathrm{e}$ 80/100.000, em cada triênio). O mesmo aconteceu quando analisadas as taxas de cada causa, com exceção das complicações anestésicas e embolias. Desse modo, reitera-se que a qualidade da atenção é de fundamental importância para a redução de mortalidade materna. As ações para sua redução devem, portanto, promover a melhoria da qualidade dos serviços, numa abordagem sistêmica, de forma a fortalecer o sistema de saúde como um todo ${ }^{12,20}$.

O presente estudo mostrou uma elevada TLM por infecção puerperal, cerca de três vezes superior nos hospitais de alto risco em relação ao outro grupo, o que pode estar associado às elevadas taxas de cesárea que ocorrem naqueles hospitais, como observado em estudo realizado em outro estado da região Sul do Brasil ${ }^{18}$. Por outro lado, as complicações anestésicas, hemorragias e embolias foram mais frequentes nos hospitais de referência para gestação de baixo risco, podendo esta situação estar relacionada a cesáreas desnecessárias praticadas também nos hospitais em que um único profissional realiza a cesárea e a anestesia. O Comitê Estadual de Prevenção da Mortalidade Materna tem identificado a falta de anestesista em diversos hospitais que prestam atendimento às gestantes de baixo risco.

$\mathrm{O}$ aborto figurou entre as principais cinco causas de mortes no período estudado, com TLM mais elevada nos hospitais de referência para gestação de alto risco, em ambos os períodos, indicando possível fragilidade na assistência prestada nesses serviços. Em estudo realizado no mesmo 
estado acerca do perfil de mulheres que morreram em decorrência do aborto, verificou-se que $88 \%$ dos óbitos eram evitáveis. $\mathrm{Na}$ análise desses óbitos apontaram-se falhas relativas à atenção profissional e institucional: inadequação das condutas profissionais e tratamento das emergências obstétricas; falta de medidas de educação em saúde no período pré-natal, principalmente às mulheres com risco reprodutivo; demora na resolução de complicações decorrentes do aborto, entre outras. Destacou-se ainda como uma das principais medidas de prevenção o aprimoramento da assistência médico-hospitalar ${ }^{22}$.

Com relação à transferência hospitalar, evidenciou-se que, para aproximadamente $64 \%$ dos casos, não ocorreu transferência hospitalar ou o óbito ocorreu no hospital no internamento inicial no primeiro triênio, e 71,6\% no segundo triênio, concluindo-se que as falhas e as causas das mortes maternas devem ser atribuídas ao hospital do primeiro internamento. Por outro lado, a não transferência pode ser consequência da falta de acesso aos hospitais de alto risco, em alguns casos. Em estudo realizado no município de Recife (PE), a frequência de transferência hospitalar foi de 38,5\% nos casos de óbitos maternos ocorridos entre 1994 e $2000^{15}$, enquanto no Paraná foi de 34,6 e 28,4\% em cada um dos períodos estudados, respectivamente.

Pode-se destacar a necessidade de ações voltadas à qualificação da assistência obstétrica, aliada a outras ações multiprofissionais e multissetoriais voltadas à minimização do conjunto de determinantes que têm marcado o perfil da mortalidade e morbidade maternas no Brasil ${ }^{4,23}$.

O Paraná conta com uma rede hospitalar para atenção à gestação de alto risco que abrange praticamente todo o estado. No entanto, a elevada taxa de evitabilidade dos óbitos maternos nestes hospitais permite inferir que provavelmente ocorram falhas nesta rede, tais como deficiência da infraestrutura, na capacitação periódica dos profissionais para tratamento das emergências obstétricas e no atendimento qualificado ao parto, incluindo a adoção de práticas baseadas em evidências científicas e humanização da assistência, que há décadas são preconizadas por organismos internacionais e pelo Ministério da Saúde do Brasil ${ }^{23,24}$.

A mortalidade materna como grave problema de saúde pública requer atenção especial, pois são mulheres no auge de suas vidas reprodutivas com potenciais de vida subitamente perdidos com a morte prematura, comprometendo suas famílias, as comunidades e a sociedade ${ }^{1,25}$. A assistência hospitalar adequada poderia ter contribuído para a evitabilidade de uma proporção significativa de óbitos maternos no Paraná, nos períodos estudados.

Conclui-se que, apesar dos avanços tecnológicos em relação à assistência à gestação, ao parto e ao puerpério (ultrassonografia, cardiotocografia, exames laboratoriais, UTIs, dentre outros recursos), as mulheres, no Brasil, estão sujeitas ao óbito por causas já controladas em muitos países, especialmente os mais desenvolvidos.

Nos estudos de caso elaborados pelos comitês, os dados e resultados obtidos permitem inferir que nos hospitais de referência para gestação de alto risco são encontradas dificuldades no manejo das complicações obstétricas. Assim sendo, reitera-se a necessidade de capacitação dos profissionais, não apenas nos serviços de alta complexidade, mas em todos os níveis de atenção à saúde.

A morbidade grave (near miss) e os óbitos maternos, dentre outros indicadores, tais como a taxa de procedimentos utilizados, muitas vezes, de modo inadequado (cesáreas, anestesias, episiotomias etc.), devem ser analisados pelos gestores e profissionais dos hospitais como uma estratégia prioritária para redução das mortes maternas evitáveis.

A utilização de protocolos e condutas preconizadas pelo Ministério da Saúde deve ser monitorada pelos serviços de auditoria do SUS e pelos comitês hospitalares, municipais e de prevenção da mortalidade materna e/ou congêneres. A responsabilização e as providências cabíveis devem ser tomadas junto aos hospitais nos casos de negligência.

Estudos detalhados para cada hospital são recomendáveis. A contratação dos hospitais de referência para atenção à gestação de baixo risco e especialmente para o alto risco gestacional deveria ser criteriosa, exigindo-se o cumprimento das portarias e dos protocolos definidos pelo Ministério da Saúde. Seria recomendável a monitoração da capacitação das equipes de saúde, dos seus indicadores e do funcionamento geral do atendimento de saúde à mulher em fase reprodutiva para que se diminuam os alarmantes níveis de mortalidade materna e suas lamentáveis e indesejáveis consequências.

\section{Referências}

1 Reis LGC, Pepe VLE, Caetano R. Maternidade segura no Brasil: o longo percurso para a efetivação de um direito. Physis (Rio J). 2011;21(3):1139-60.

2. Lozano R, Wang H, Foreman KJ, Rajaratnam JK, Naghavi M, Marcus JR, et al. Progress towards Millennium Development Goals 4 and 5 on maternal and child mortality: an updated systematic analysis. Lancet. $2011 ; 378(9797): 1139-65$.
3. World Health Organization. UNICEF. UNFPA. World Bank [Internet]. Trends in maternal mortality: 1990 to 2008. Geneva: WHO; 2010 [cited 2012 Set 7]. Available from: <http://whqlibdoc. who.int/publications/2010/9789241500265_eng.pdf>

4. Soares VMN, Souza KV, Freygang TC, Correa V, Saito MR. Maternal mortality due to pre-eclampsia/eclampsia in a state in Southern Brazil. Rev Bras Ginecol Obstet. 2009;31(1 1):566-73. 
5. Morse ML, Fonseca SC, Barbosa MD, Calil MB, Eyer FPC. Mortalidade materna no Brasil: o que mostra a produção científica nos últimos 30 anos? Cad Saúde Pública. 2011 ;27(4):623-38.

6. Luz AG, Tiago DB, Silva JCG, Amaral E. Severe maternal morbidity at a local reference university hospital in Campinas, São Paulo, Brazil. Rev Bras Ginecol Obstet. 2008;30(6):281-6.

7. Brasil. Ministério da Saúde. Secretaria de Atenção à Saúde. Departamento de Ações Programáticas Estratégicas. Manual dos comitês de mortalidade materna. 3a ed. Brasília (DF): Editora do Ministério da Saúde; 2009.

8. Matias JP, Parpinelli MA, Nunes MKV, Surita FGC, Cecatti JG. Comparison of two methods for the investigation of maternal mortality in a municipality of the Brazilian Southeast. Rev Bras Ginecol Obstet. 2009;31(11):559-65.

9. Soares VMN, Martins AL. A trajetória e experiência dos comitês de prevenção da mortalidade do Paraná. Rev Bras Saúde Matern Infant. 2006;6(4):453-60.

10. Victora CG, Aquino EM, Leal MC, Monteiro CA, Barros FC, Szwarcwald CL. Maternal and child health in Brazil: progress and challenges. Lancet. $2011 ; 377(9780)$ : 1863-76.

11. Brasil. Presidência da República [Internet]. Objetivos de desenvolvimento do milênio: relatório nacional de acompanhamento. Brasília (DF): IPEA; 2010 [citado 2012 Set 7]. Disponível em: <http://mds.gov. $\mathrm{br} /$ bolsafamilia/arquivos/relatorio-nacional-de-acompanhamento. pdf/download>

12. Souza JP. Maternal mortality in Brazil: the need for strengthening health systems. Rev Bras Ginecol Obstet. 201 1;33(10):273-9.

13. Soares VMN, Azevedo EMM, Watanabe TL. Subnotificação da mortalidade materna no estado do Paraná, Brasil: 1991-2005. Cad Saúde Pública. 2008;24(10):2418-26.

14. Organização Mundial de Saúde. Cid-10: classificação estatística internacional de doenças e problemas relacionados à saúde. São Paulo: Edusp; 1997.

15. Costa AAR, Ribas MSSS, Amorim MMR, Santos LC. Maternal mortality in Recife. Rev Bras Ginecol Obstet. 2002;24(7):455-62.
16. Reis ZSN, Pereira AC, Correia RJC, Freitas JAS, Cabral ACV, Bernardes J. Analysis of maternal and child health indicators: the parallel between Portugal and Brazil. Rev Bras Ginecol Obstet. $2011 ; 33(9): 234-9$.

17. United Nations [Internet]. The millennium development goals report. New York: United Nations; 2011 [cited 2012 Set 7]. Available from: <http://www.un.org/millenniumgoals/11_MDG\%20 Report_EN.pdf>

18. Ramos JGL, Martins-Costa S, Vettorazzi-Stuczynski J, Brietzke E. Maternal mortality at a tertiary hospital in Rio Grande do Sul - Brazil: a twenty-year study. Rev Bras Ginecol Obstet. 2003;25(6):43 1-6.

19. Cecatti JG, Calderón IMP. Beneficial interventions during delivery for the prevention of maternal mortality. Rev Bras Ginecol Obstet. $2005 ; 27(6): 357-65$

20. Cross S, Bell JS, Graham WJ. What you count is what you target: the implications of maternal death classification for tracking progress towards reducing maternal mortality in developing countries. Bull World Health Organ. 2010;88(2):147-53.

21. Andrade ATL, Guerra MO, Andrade GN, Araújo DAC, Souza JP. Maternal mortality: 75 years of observations in a teaching maternity hospital. Rev Bras Ginecol Obstet. 2006;28(7):380-7.

22. Souza KV, Almeida MRCB, Soares VMN. Perfil da mortalidade materna por aborto no Paraná: 2003-2005. Esc Anna Nery Rev Enferm. 2008;12(4):741-9

23. Amaral E, Luz AG, Souza JPD. The severe maternal morbidity for the qualification of care: utopia or necessity? Rev Bras Ginecol Obstet. 2007;29(9):484-9.

24. Rocha JA, Novaes PB. Uma reflexão após 23 anos das recomendações da Organização Mundial da Saúde para parto normal. Femina. 2010;38(3): $119-26$.

25. Brasil. Ministério da Saúde. Secretaria de Atenção à Saúde. Departamento de Ações Programáticas Estratégicas. Estudo da mortalidade de mulheres de 10 a 49 anos, com ênfase na mortalidade materna: relatório final. Brasília (DF): Ministério da Saúde; 2006. 